\title{
Association between the expression of chemokine receptors CCR7 and CXCR3, and lymph node metastatic potential in lung adenocarcinoma
}

\author{
SHINICHI MAEKAWA ${ }^{1}$, AKINORI IWASAKI ${ }^{1}$, TAKAYUKI SHIRAKUSA ${ }^{1}$, \\ TAKEHITO KAWAKAMI ${ }^{2}$, JUN YANAGISAWA ${ }^{1}$, TOSHIHIRO TANAKA ${ }^{3}$, HIROTOMO SHIBAGUCHI ${ }^{3}$, \\ TESTUSHI KINUGASA ${ }^{3}$, MOTOMU KUROKI ${ }^{3}$ and MASAHIDE KUROKI ${ }^{3}$
}

Departments of ${ }^{1}$ Thoracic Surgery, ${ }^{2}$ Pathology, ${ }^{3}$ Biochemistry, Faculty of Medicine, Fukuoka University, Japan

Received January 30, 2008; Accepted March 3, 2008

\begin{abstract}
Chemokines and their receptors are essential for leukocyte trafficking, and are also involved in cancer metastasis to specific organs. Although the migration of tumor cells into the lymph nodes is an important aspect of cancer, the processes involved are poorly understood. Chemokine receptors CCR7 and CXCR3 have been shown to play an important role in tumor cell migration and lymph node metastasis. Therefore, the assessment of chemokine receptor expression on lung adenocarcinomas may improve the prediction of the spread of this carcinoma to the lymph nodes. In this study, we examined the expression and function of these two chemokine receptors (CCR7 and CXCR3) in lung adenocarcinoma. By using flow cytometry, they were detected in all of the lung adenocarcinoma cell lines examined. In the chemotaxis assays, A549 cells exhibited CCL21-induced migration, which was significantly suppressed by neutralizing anti-CCR7 antibody. The CXCL10-induced migration of A549 cells was also significantly suppressed by neutralizing anti-CXCR3 antibody. In clinical lung adenocarcinoma samples, we found the expression of CCR7 and CXCR3 in 65 and $90 \%$ cases, respectively, most of which had lymph node metastasis. Importantly, the expression of CCR7 was significantly associated with lymph node metastasis, although the expression of CXCR3 was not. These results suggest that the activation of CCR7 and CXCR3 with their ligands preferentially stimulates lung adenocarcinoma metastasis to the draining lymph nodes.
\end{abstract}

Correspondence to: Dr S. Maekawa, Department of Thoracic Surgery, Faculty of Medicine, Fukuoka University, 45-1, 7-chome Nanakuma, Jonan-ku, Fukuoka 814-0180, Japan

E-mail: nwnkr090@ybb.ne.jp

Key words: CCR7, CXCR3, lung adenocarcinoma, lymph node metastasis

\section{Introduction}

Non-small cell lung cancer (NSCLC) is the leading cause of cancer-related death worldwide, and its incidence and mortality rate continue to rise. The poor prognosis of NSCLC is mediated in part by the specific and aggressive metastatic pattern of the primary cancer cells to regional lymph nodes, contralateral lung, brain, liver, adrenal glands and the bone marrow (1-4). The preference for the cells to metastasize to specific organs may depend on a variety of factors $(5,6)$. According to Liotta et al (7), while all the migrating carcinoma cells have the ability to invade any tissue, they only metastasize if all requirements for their growth are met. Other researchers have suggested that tissue-specific adhesion molecules on endothelial cells select migrating carcinoma cells which attach to the adhesion molecules, resulting in the development of a premetastatic nucleus of cells (8). Another theory proposes that chemoattractants, produced by stromal or immune cells, attract invasive cancer cells to tissues where they have potential for secondary growth (9). Previous studies have shown the involvement of chemokine receptors in cancer metastasis (10-12).

The chemokine family are one of small molecular weight (8-14 kDa) chemotactic cytokines that bind to G-proteincoupled heptahelical receptors, an action which plays a pivotal role in the regulation of leukocyte trafficking and their extravasation through the luminal surface of endothelial cells into sites of tissue inflammation (13-15). Chemokine ligands CCL19 and CCL21, which are constitutively expressed by lymph node cells and other immune cells, share a common chemokine receptor, CCR7 (11). It has been reported that one of these CCR7 ligands (CCL21) can bind to, and activate, the chemokine receptor CXCR3 in mice (16).CCL19 and CCL21 share CXCR3 with two other chemokine ligands CXCL9 and CXCL10, which are also constitutively expressed by lymph nodes $(17,18)$. On the other hand, the chemokine receptor CCR7 is essential for the recruitment to the lymph nodes of naïve $\mathrm{T}$, certain memory $\mathrm{T}$ and mature dendritic cells, and for the retention of Th1 lymphocytes within lymph nodes $(19,20)$. Similarly, the chemokine receptor CXCR3 plays a central role in the recruitment of plasmacytoid dendritic cell precursors, monocytes and natural killer cells to the inflamed lymph nodes 
(21-23). Regarding the direct role of chemokines in lymph node metastasis, previous reports suggest a critical role for the chemokine receptors CCR7 and CXCR3 in the metastasis of melanoma and breast cancer $(12,18,24)$.

In this study, we demonstrate that CCR7 and CXCR3 are expressed by human lung adenocarcinoma cell lines and primary $\mathrm{T} 1$ lung adenocarcinoma tissues and that they play a key role in lung adenocarcinoma metastasis to the lymph nodes. Our results also suggest that CCR7 and CXCR3 are potential novel therapeutic targets that suppress lymph node metastasis in lung adenocarcinoma.

\section{Materials and methods}

Cell lines. The lung adenocarcinoma cell lines A549, NCIH838, NCI-H1395 and NCI-H1650 were obtained from the American Type Culture Collection (Manassas, VA, USA). The lung adenocarcinoma cell lines HLC-1 and RERF-LC-KJ were obtained from RIKEN cell bank (Tsukuba, Ibaragi, Japan). Cells were cultured in RPMI-1640 medium (Sigma-Aldrich, St. Louis, MO, USA), supplemented with $10 \%$ heat-inactivated fetal bovine serum (FBS) (Bio Whittaker, Walkersville, MD, USA), $100 \mathrm{U} / \mathrm{ml}$ penicillin $\mathrm{G}$ and $100 \mathrm{mg} / \mathrm{ml}$ streptomycin (Invitrogen, Carlsbad, CA, USA).

Tissue samples. From January 2004 to January 2006, 281 consecutive patients with a clinical diagnosis of NSCLC underwent surgical resection at the Department of Thoracic Surgery, Fukuoka University Hospital, Japan. Tissue samples were randomly obtained from 20 of these patients with a primary $\mathrm{T} 1$ ( $<3 \mathrm{~cm}$ in diameter) lung adenocarcinoma: 10 cases with and 10 cases without lymph node metastases. Written informed consent for the molecular analysis of the surgical sample was obtained from each patient. A histological diagnosis was confirmed for each specimen. All data concerning the histology, lymph node metastasis and disease stage were obtained from the patient clinical and pathological records. The disease stage was classified according to the criteria proposed by the tumor-node-metastasis (TNM) classification (25).

Flow cytometry. The cells were fixed by Inside Stain Kit (Miltenyi Biotec, Bergisch Gladbach, Germany), according to the manufacturer's instructions. Briefly, $1 \times 10^{5}$ cells were suspended in a mixture of $250 \mu 1$ phosphate-buffered saline (PBS) containing $0.5 \%$ bovine serum albumin, 2 mM EDTA, $0.1 \% \mathrm{NaN}_{3}$ and $250 \mu \mathrm{l}$ Inside Fix in a $2.0 \mathrm{ml}$ Eppendorf tube. After incubation for $20 \mathrm{~min}$ at room temperature, the cells were washed twice in PBS. The cells reacted with antihuman CCR7 monoclonal antibody (R\&D Systems, Minneapolis, MN, USA) and anti-human CXCR3 monoclonal antibody (R\&D Systems) in Inside Perm for $20 \mathrm{~min}$ at room temperature. A mouse $\mathrm{IgG}_{1}$ antibody, MOPC21 (Beckman Coulter, Fullerton, CA, USA), was used as the negative control. After adding $1 \mathrm{ml}$ of Inside Perm, the cells were centrifuged, washed in PBS and incubated with phycoerythrin (PE)-conjugated goat polyclonal mouse IgG antibody (Abcam, Cambridge, MA, USA). After washing, the cells were analyzed on a FACScan cytometer using CellQuest software.
Chemotaxis assay. Cell migration was determined using a modified Boyden chamber assay as previously described (26). Briefly, the Chemicon QCM 96-well cell migration assay kit (Chemicon, Temecula, CA, USA) with an $8-\mu \mathrm{m}$ pore size was run three times. Cells were briefly trypsinized and adjusted to $1 \times 10^{6}$ cells $/ \mathrm{ml}$ in RPMI-1640 medium, which contained $1 \%$ FBS (Bio Whittaker). Ligands of the chemokine receptors CCR7, CCL21 and CCL19 (R\&D Systems), and a ligand of the chemokine receptors CXCR3 and CXCL10 (R\&D Systems) were dissolved in the medium to various concentrations. After the different concentrations of CCL21, CCL19 and CXCL10 had been added to the lower chamber as attractants, the cells $\left(1 \times 10^{3}\right.$ cells $\left./ \mathrm{ml}\right)$ were applied to the upper chamber on the top of the membrane. In neutralization studies, the cells were incubated with the various concentrations of either anti-human CCR7 or anti-human CXCR3 antibody (R\&D Systems) for $2 \mathrm{~h}$ at $4^{\circ} \mathrm{C}$ before being used in the chemotaxis assays. After $18 \mathrm{~h}$ of incubation at $37^{\circ} \mathrm{C}$ in a $\mathrm{CO}_{2}$ incubator, the migratory cells on the bottom of the inserted membrane were dissociated from it by incubation with a cell detachment buffer. The cells were subsequently lysed and stained with CyQuant GR dye (Chemicon), which exhibits strong fluorescence enhancement when bound to cellular nucleic acids, and the fluorescence was measured with a fluorescence plate reader with a 480/520 $\mathrm{nm}$ filter set.

Time-lapse images of the lung adenocarcinoma cell line during chemotaxis were obtained using EZ-TAXIScan ${ }^{\mathrm{TM}}$ (GE Healthcare, Tokyo, Japan) as previously described (27). The lung adenocarcinoma cells $\left(5 \times 10^{3}\right.$ cells $\left./ \mathrm{ml}\right)$ were injected into one of the two compartments through a hole connected to that compartment. To adjust for the position of the cells, the medium was drawn out through the hole connected to the opposite compartment. This process was stopped when the cells were aligned in the channel close to the edge. Aliquots of various concentrations of CCL21 $(1 \mu \mathrm{l})$ were then injected into the other compartment to initiate chemotaxis and monitoring began. The images of the channels were recorded, digitally, channel by channel onto a computer hard disk at time-lapse intervals with a high performance lens connected to a CCD camera and a coaxial episcopic illumination system.

Immunohistochemistry. Immunohistochemical staining using the dextran polymer method was used to assess CCR7 and CXCR3. Briefly, paraffin-embedded $5 \mu \mathrm{m}$ sections obtained from surgical specimens fixed in $10 \%$ formalin were de-waxed in xylene and dehydrated through graded concentrations of ethanol. Endogenous peroxidase was blocked by incubation in a peroxidase blocking solution (S2023, Dako Cytomation, Kyoto, Japan). Antigen retrieval was performed for $10 \mathrm{~min}$ at $95^{\circ} \mathrm{C}$ in a microwave. After these procedures, the sections were washed in TBST $(50 \mathrm{mM}$ Tris- $\mathrm{HCl}$ buffer, $\mathrm{pH} 7.6$, including $0.1 \%$ Tween-20 and $0.15 \mathrm{M} \mathrm{NaCl}$ ) for $5 \mathrm{~min}$, followed by incubation with the primary antibodies for CCR7 and CXCR3 at 1:100 dilutions for $30 \mathrm{~min}$. After washing three times with TBST, the sections were incubated with Envision $^{+}$(K4000, Dako Cytomation) for $30 \mathrm{~min}$ in a humidified box at room temperature. The sections were washed again three times in TBST for $5 \mathrm{~min}$, developed with $\mathrm{DAB}^{+}$(K3467, Dako Cytomation) for $5 \mathrm{~min}$ and counterstained with hematoxylin solution for $1 \mathrm{~min}$. 
A
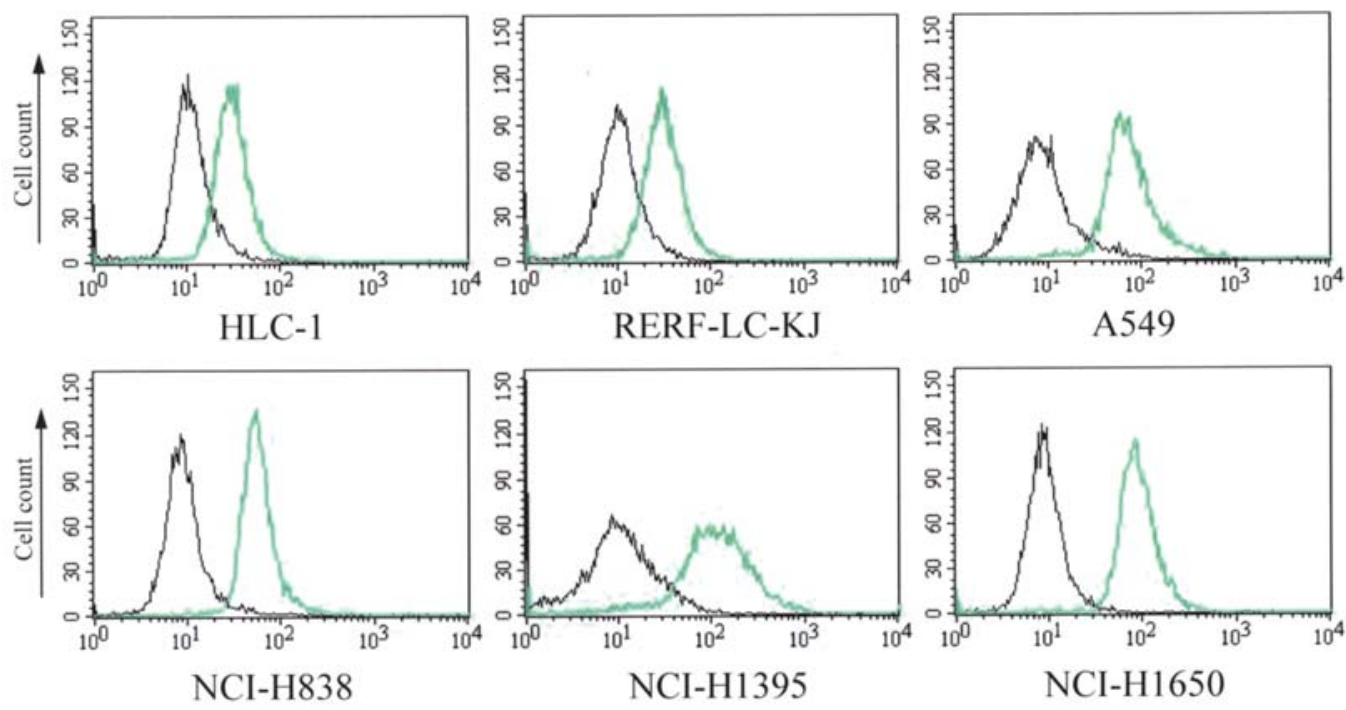

B
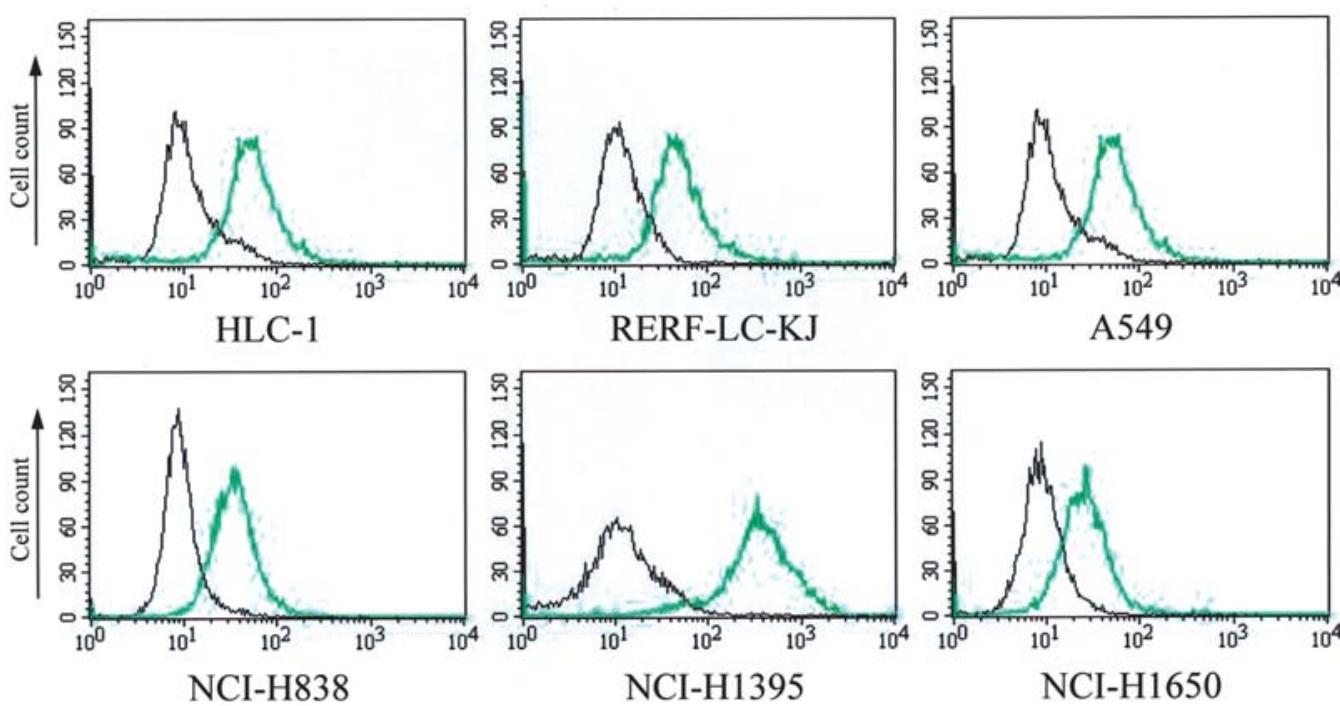

Figure 1. The expression of chemokine receptors CCR7 and CXCR3 in human lung adenocarcinoma cell lines. The cell surface expression of CCR7 (A) and CXCR3 (B) were detected in HLC-1, RERF-LC-KJ, A549, NCI-H838, NCI-H1395 and NCI-H1650 by flow cytometry using a monoclonal anti-CCR7 or anti-CXCR3 antibody, respectively. The green lines represent cells stained with a monoclonal anti-CCR7 or anti-CXCR3 antibody, whereas the black lines show cells stained with the isotype-matched control monoclonal antibody.

We interpreted a sample as positive for CCR7 or CXCR3 when $>10 \%$ of the tumor cells were stained with either antibody.

Statistical analysis. Data were expressed as the mean \pm standard deviation (SD) of repeated assays. Statistical differences between the two groups were evaluated using the $\chi^{2}$ test or Student's t-test. The calculations were performed with the software Statview ${ }^{\mathrm{TM}}$ (Abacus Concepts, Berkley, CA, USA). Values of $\mathrm{p}<0.05$ were considered significant.

\section{Results}

Expression of chemokine receptors $C C R 7$ and $C X C R 3$ in lung adenocarcinoma cell lines. We performed flow cytometry to analyze the expression of CCR7 and CXCR3 proteins in six human lung adenocarcinoma cell lines. As shown in Fig. 1, the lung adenocarcinoma cell lines (HLC-1, RERF-LC-KJ, A549, NCI-H838, NCI-H1395 and NCI-
H1650) expressed CCR7 (panel A) and CXCR3 (panel B). Since A549 cells exhibited the same levels of CCR7 and CXCR3 expression, we used this cell line as our model of CCR7- and CXCR3-expressing lung adenocarcinoma in the subsequent experiments.

CCR7- and CXCR3-mediated cellular responses of A549 cells in vitro. To determine whether CCR7 expressed by lung adenocarcinoma cells was functional, we examined the chemotactic responses of A549 cells to CCL21. As shown in Fig. 2A, A549 cells showed a significant chemotactic response to CCL21 in a typical bell-shaped dose-dependent manner. Furthermore, the neutralizing anti-CCR7 antibody significantly suppressed the migratory response in a dose-dependent manner (Fig. 2A). It has been reported that one of the CCR7 ligands, CCL21, can bind to and activate CXCR3 in mice (16), and that CCL21 is a functional ligand for endogenously expressed CXCR3 (28). Therefore, we investigated whether the cocktail of the neutralizing anti-CXCR3 and anti-CCR7 
A

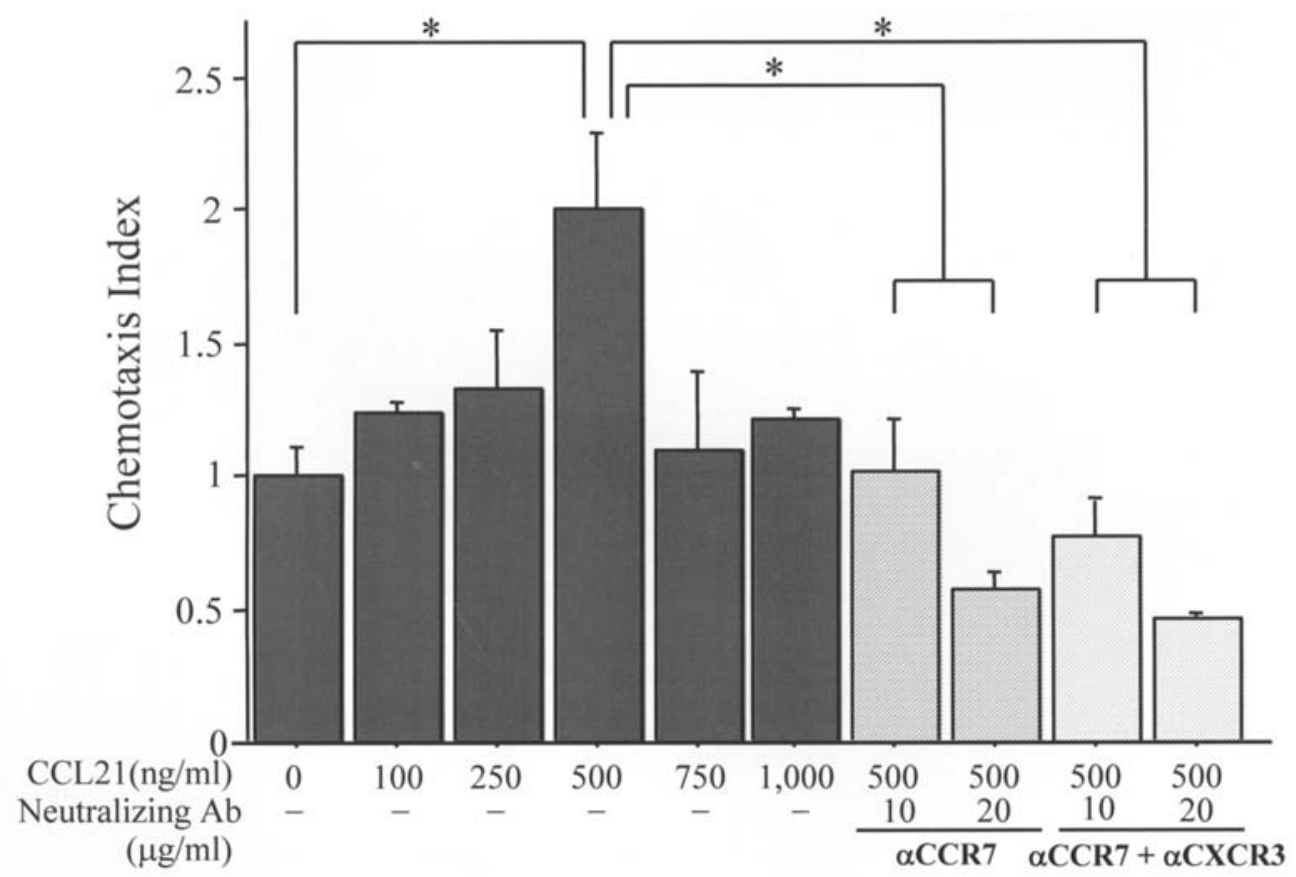

B

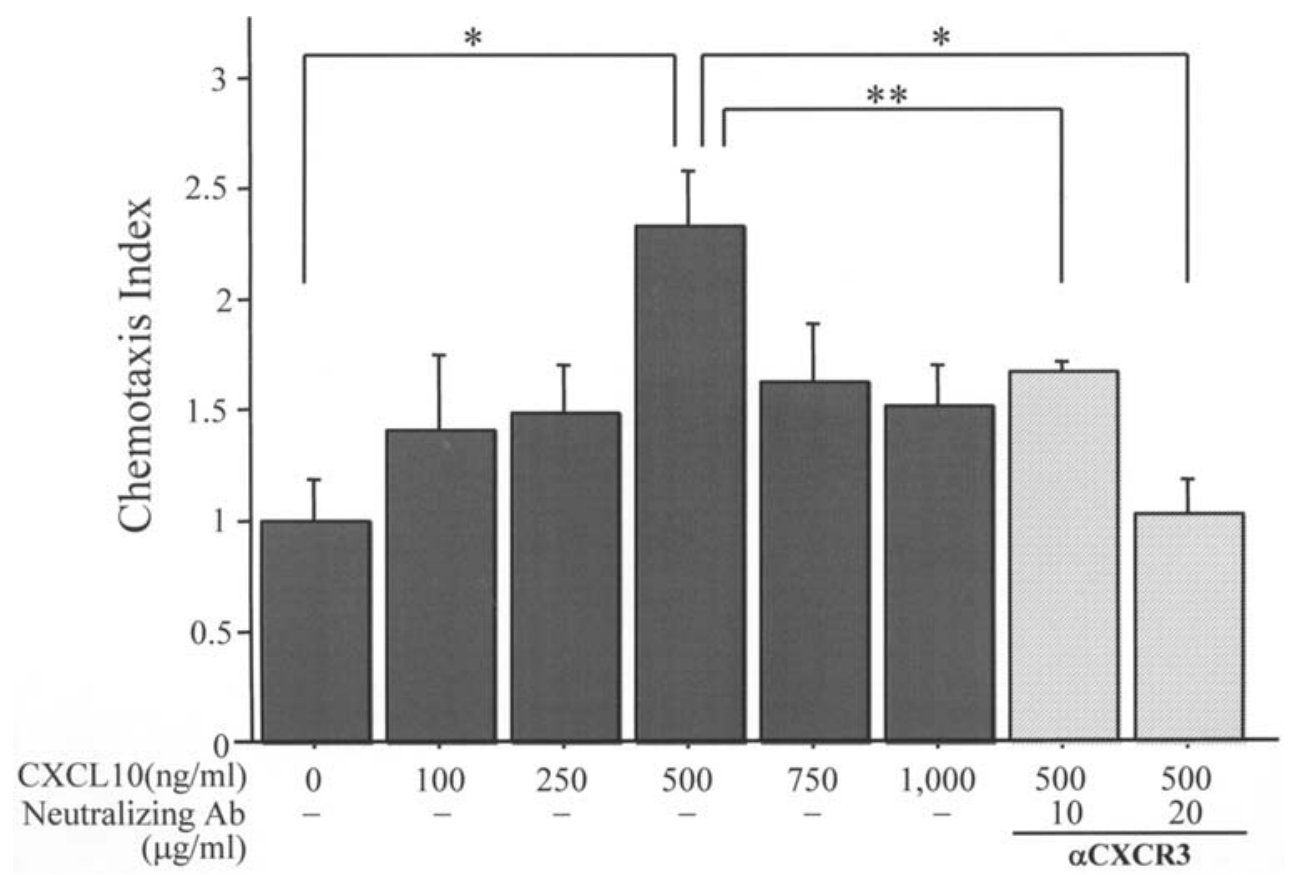

Figure 2. Activation of CCR7 and CXCR3 on A549 cells with their ligands. Chemotaxis assays in modified transwell chambers. (A) Dose-dependent chemotactic responses to CCL21 and effect of the neutralizing anti-CCR7 antibody on CCR7-mediated chemotaxis or effect of the neutralizing anti-CCR7 and anti-CXCR3 antibodies on CXCR3-mediated chemotaxis. (B) Dose-dependent chemotactic responses to CXCL10 and effect of the neutralizing antiCXCR3 antibody on CXCR3-mediated chemotaxis. The bars are the mean \pm SD ( ${ }^{*} \mathrm{p}<0.01$ and ${ }^{* *} \mathrm{p}<0.05$ by Student's t-test).

antibodies inhibit the CCL21-induced migration. Fig. 2A shows that the cocktail suppressed the CCL21-induced migration more than the neutralizing anti-CCR7 antibody alone, although the differences were not statistically significant. Along with CCL21, we examined the chemotactic responses of A549 cells to CCL19, but significant differences were not observed (data not shown). We then examined the chemotactic responses of A549 cells to CXCL10. As shown in Fig. 2B, A549 cells showed significant chemotactic responses to CXCL10 in a bell-shaped dose-dependent manner. The neutralizing anti-CXCR3 antibody significantly inhibited the migratory response in a dose-dependent manner (Fig. 2B).

Furthermore, the cells were imaged every 3 min by using EZ-TAXIScan. Fig. 3 shows images of the migration of A549 cells following exposure to CCL21, as observed by EZ-TAXIScan. The chemokine-stimulated A549 cells began to migrate one by one towards the other end of the channel where the concentration of the chemokine was highest.

CCR7 and CXCR3 expression and the correlation between chemokine receptor expression levels and lymph node 
A

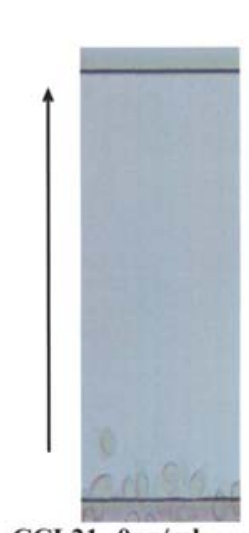

CCL21 0ng/ml

B

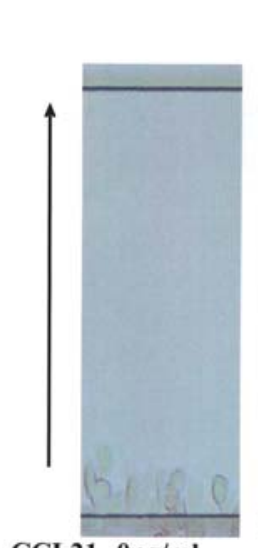

CCL21 0ng/ml

C

D

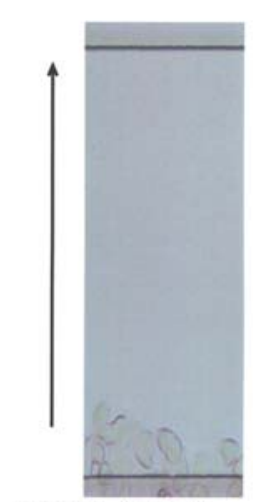

CCL21 0ng/ml

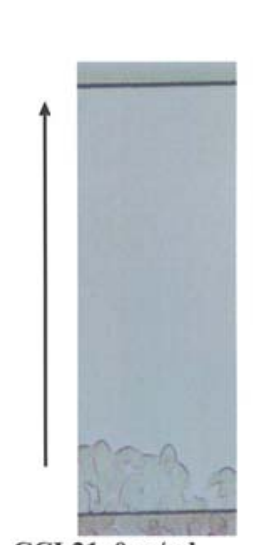

CCL21 0ng/ml

\section{$\underline{0 \mathrm{hr}}$}

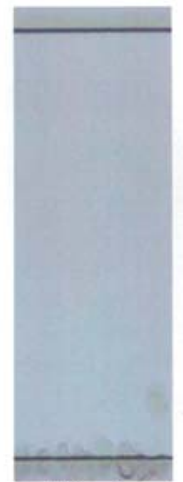

$100 \mathrm{ng} / \mathrm{ml}$

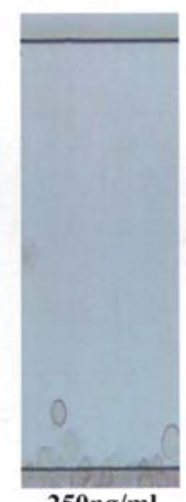

$250 \mathrm{ng} / \mathrm{ml}$

\section{$\underline{1 \mathrm{hr}}$}

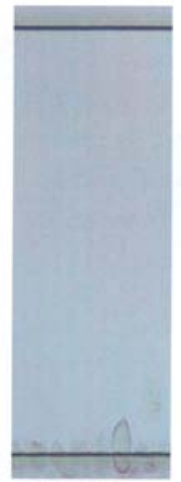

$100 \mathrm{ng} / \mathrm{ml}$

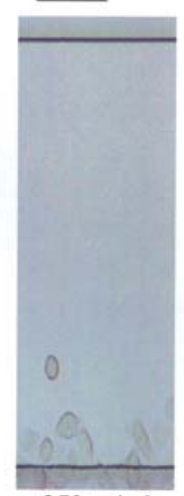

$250 \mathrm{ng} / \mathrm{ml}$

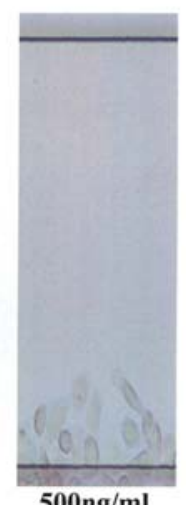

$500 \mathrm{ng} / \mathrm{ml}$

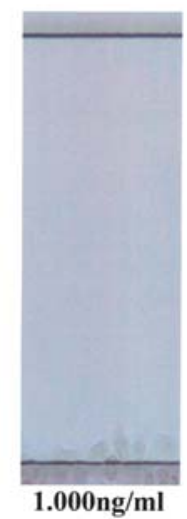

$\underline{3 \mathrm{hr}}$

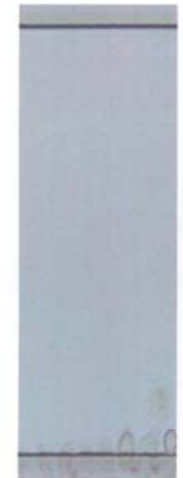

$100 \mathrm{ng} / \mathrm{ml}$

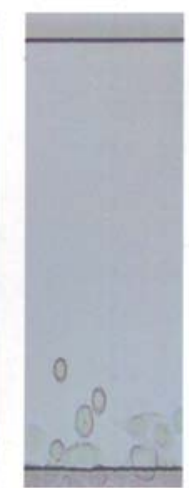

$250 \mathrm{ng} / \mathrm{ml}$

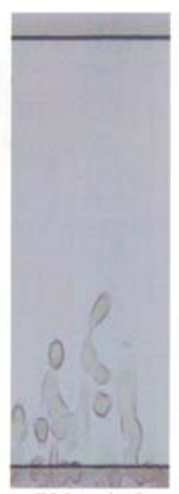

$500 \mathrm{ng} / \mathrm{ml}$

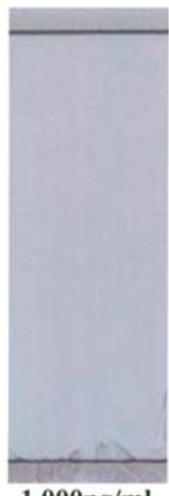

$1.000 \mathrm{ng} / \mathrm{ml}$

\section{$\underline{5 \mathrm{hr}}$}

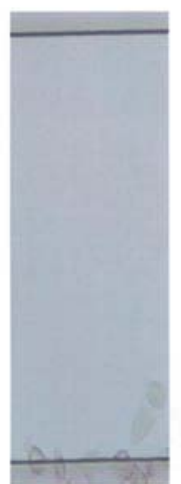

$100 \mathrm{ng} / \mathrm{ml}$

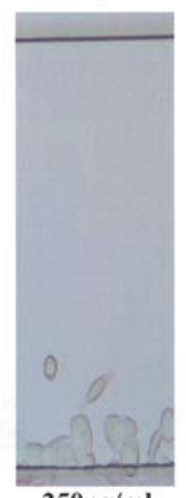

$250 \mathrm{ng} / \mathrm{ml}$

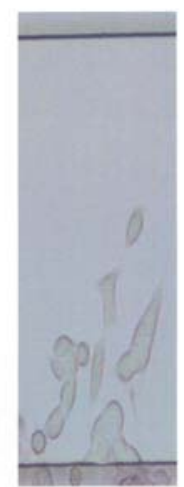

$500 \mathrm{ng} / \mathrm{ml}$

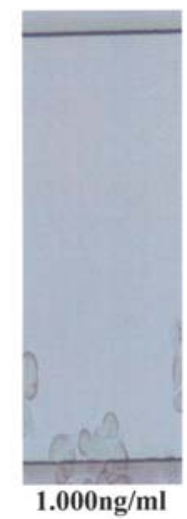

Figure 3. A549 cell chemotaxis towards a concentration gradient of various concentrations CCL21. Images of A549 cell chemotaxis were filmed every 3 min using EZ-TAXIScan. A concentration gradient of CCL21 in the channel formed from the top to the bottom of the figure. Representative images are shown of A549 cell migration at the start 0 hours (A) and then 1 (B), 3 (C) and $5 \mathrm{~h}$ (D) after interaction with CCL21. 
A

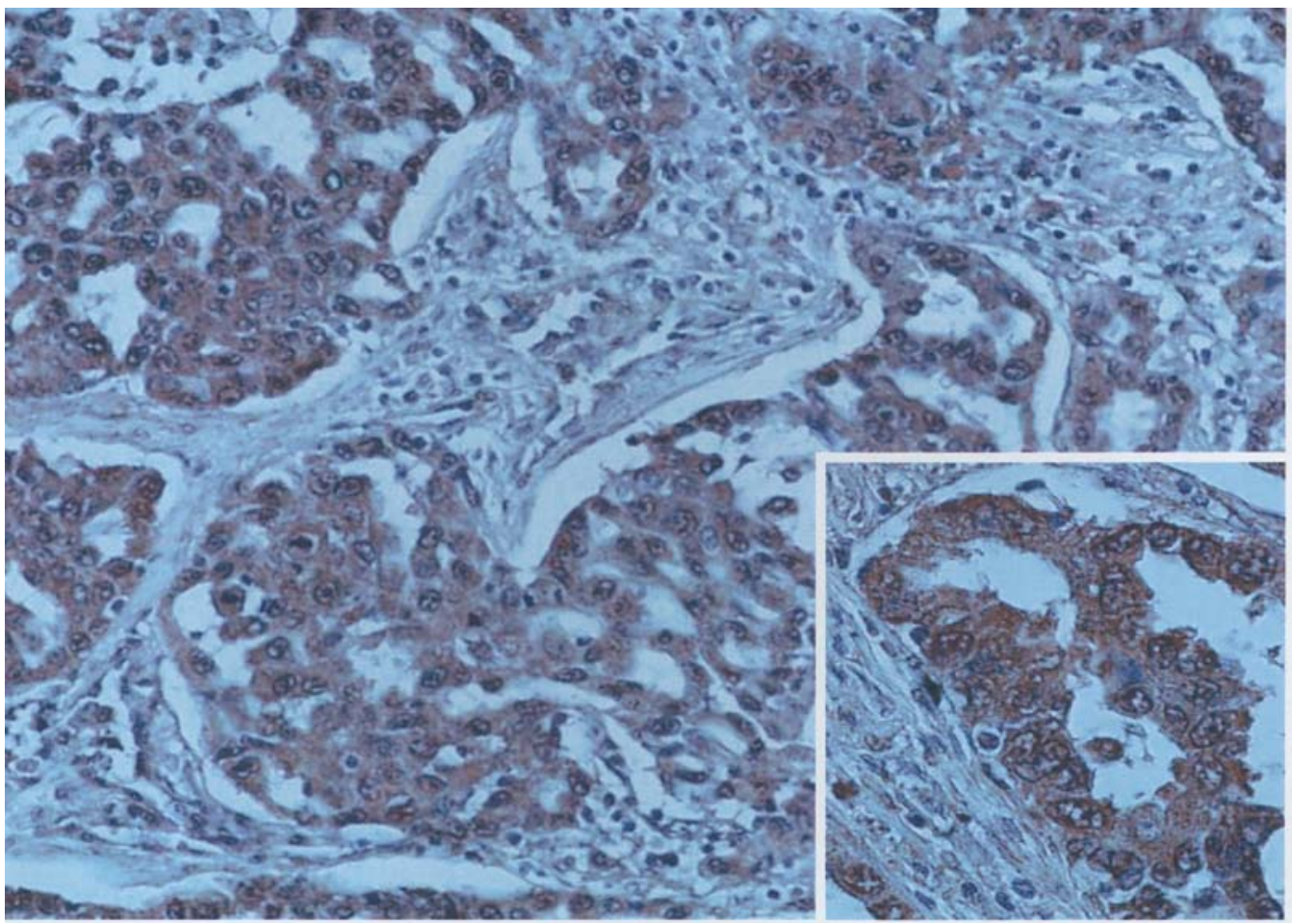

B

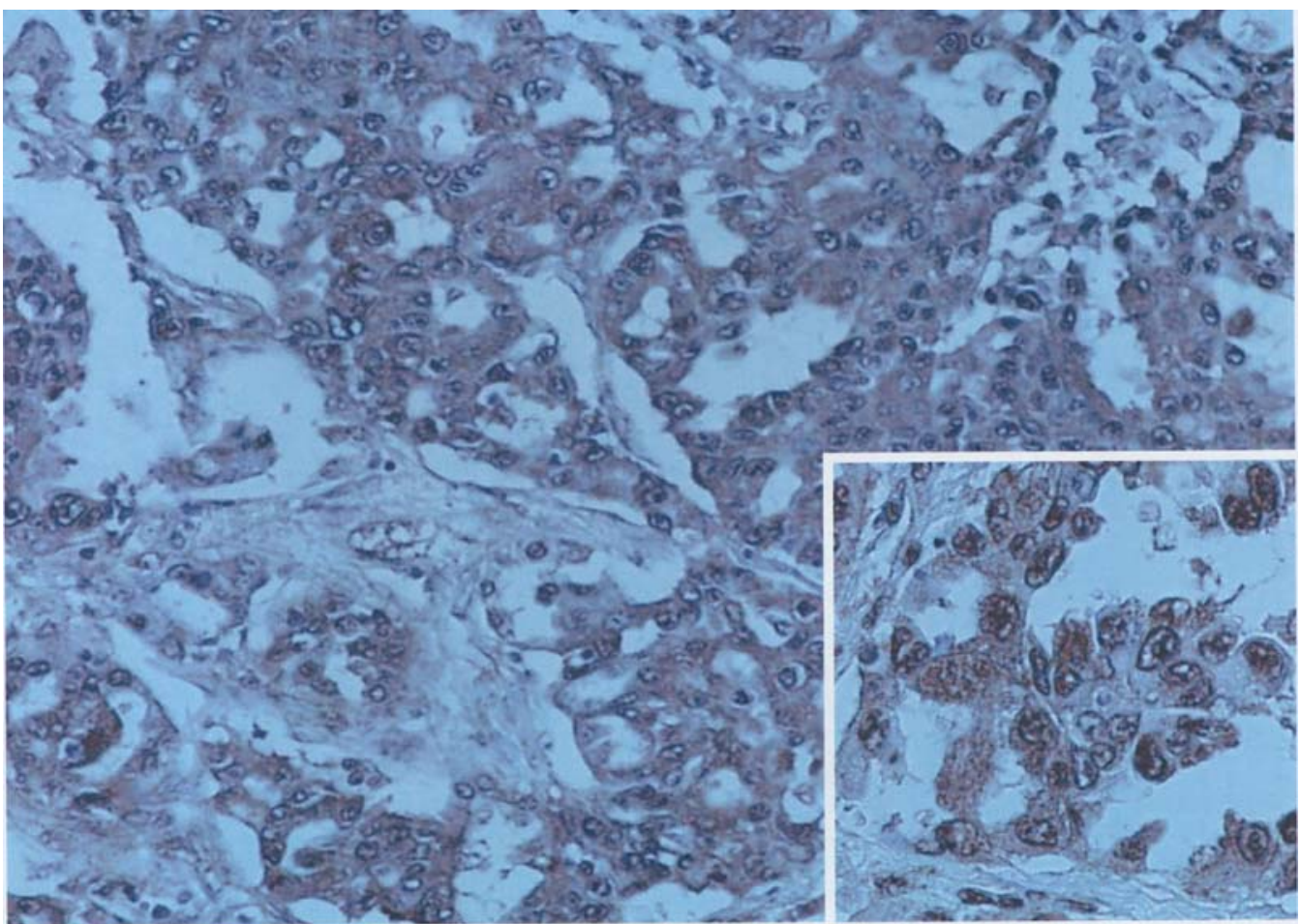

Figure 4. Immunohistochemical staining of CCR7 (A) and CXCR3 (B) expression in the primary human T1 lung adenocarcinoma. Primary lung adenocarcinoma tissues were immunostained with anti-CCR7 or anti-CXCR3 antibody. CCR7 and CXCR3 staining were detected in the membrane and cytoplasm of cancer cells. The original magnification was x100. The insets show higher magnifications of the boxed areas (x200).

metastasis in clinical samples. To examine the clinical relevance of the expression of CCR7 and CXCR3, we immunohistochemically examined specimens from 20 lung adenocarcinoma patients. We found that 13 of the samples (65.0\%) expressed CCR7 in cancerous epithelial cells, whereas $18(90.0 \%)$ expressed CXCR3 and 2 samples expressed neither CCR7 nor CXCR3. The CCR7 and CXCR3 proteins were detected in the plasma membrane and cytoplasm of the cancer cells (Fig. 4A and B) but not in the normal lung epithelial cells. The expression of CCR7 was significantly associated with lymph node metastasis (Table I), whereas there was no significant correlation between the expression of CXCR3 and lymph node metastasis. Cancer cells metastasized to lymph nodes in 9 out of the 13 (69.2\%) cases 
Table I. Significant correlations between CCR7 expression in lung adenocarcinoma and lymph node metastasis.

\begin{tabular}{lcc}
\hline Lymph node metastasis & \multicolumn{2}{c}{ CCR7 } \\
\cline { 2 - 3 } & $+(\mathrm{n}=13)$ & $-(\mathrm{n}=7)$ \\
\hline Positive & $9^{\mathrm{a}}$ & 1 \\
Negative & 4 & 6 \\
\hline
\end{tabular}

Immunohistochemical staining of CCR7 was performed in 20 samples of lung adenocarcinoma with $(\mathrm{n}=10)$ or without $(\mathrm{n}=10)$ lymph node metastasis, ${ }^{\mathrm{a}} \mathrm{p}<0.05, \chi^{2}$ test and two-sided.

that expressed CCR7 but did not in 1 out of the 7 (14.3\%) cases that showed no CCR7 expression.

\section{Discussion}

In this article we examined the CCR7- and CXCR3-mediated cellular responses of lung adenocarcinoma cell lines and the correlation between lymph node metastasis and the expression of CCR7 or CXCR3 in human primary T1 lung adenocarcinoma. Takanami et al (10) previously demonstrated that, in NSCLC patients, the CCR7 mRNA expression was significantly associated with lymph node metastasis, tumor stage, lymphatic invasion and the expression of CCR7 and CXCR4 proteins. However, our present report demonstrated that CXCR3, as well as CCR7, are expressed in lung adenocarcinoma cell lines, thus suggesting that CCR7 and CXCR3 play a role in lung adenocarcinoma metastasis to lymph nodes. The expression of CCR7 and CXCR3 was found in the six lung adenocarcinoma cell lines tested. To characterize their role in the migration of cancer cells, we used the migration assay and the real-time chemotaxis assay system EZTAXIScan, which is able to visualize cell migration. Results obtained with this assay suggested a functional interaction between the chemokine receptor CCR7 and its ligand CCL21 in A549 cells. Tumor cells use chemokine-mediated mechanisms, such as the regulation of leukocyte trafficking, during the process of metastasis (29). Cancerous epithelial cells undergo clonal proliferation, invade local tissues, induce angiogenesis and express CCR7 on their surface (29). Two constitutively expressed chemokines, CCL21 and CCL19, have been shown to be abundant in lymph nodes (14) and are, therefore, likely candidates for attracting CCR7expressed tumor cells to the regional lymph nodes. Previous studies $(30,31)$ showed that CCL21 is produced by lymphatic endothelial cells as well as lymph nodes. The migration of malignant cells from peripheral tissue into the lymphatic system may be an active process mediated by the interaction between CCL21 and CCR7. Our results from the in vitro experiments indicated that the functional CCR7 is expressed in lung adenocarcinoma cells and may be relevant to the process by which these cells preferentially migrate to lymph nodes where they will subsequently metastasize. Moreover, we showed that $65 \%$ of lung adenocarcinoma tissues expressed CCR7 and found a significant correlation between the expression of CCR7 and lymph node metastasis. These findings were in keeping with those of other studies $(30,32,33)$.

Studies have found that the chemokine receptor CXCR3 was highly expressed in melanoma and breast carcinomas and the interaction between this receptor and its ligand, CXCL10, resulted in chemotaxis or the directed migration of tumor cells from their primary site via the circulation to preferential sites of metastasis $(12,18,24)$. In the present study, we also demonstrated that CXCL10 induced lung cancer cell migration. Importantly, CCR7 and CXCR3 are involved in the recruitment and patterning of several types of immune cells to the lymph nodes, suggesting that tumor cells have acquired these receptors and are controlled by their ligands in a manner similar to the immune cell interactions. We have confirmed herein that the CCR7 ligand CCL21 is also functional for CXCR3 in human lung adenocarcinoma cells. Although in this study there was no significant correlation between the expression of CXCR3 in the primary lung adenocarcinoma tissues and lymph node metastasis, our present findings suggest that CXCR3 is involved in the metastasis of lung adenocarcinoma cells.

Notably, the level of expression of CCR7 and CXCR3 proteins was higher in the lung cancer cells that invaded the stroma than in those that did not, and CXCR3 protein was likely to be expressed earlier than the CCR7 protein in the process of cancer development (data not shown). Kawakami et al (34) demonstrated that the degree of stromal invasion correlates with prognosis even in pT1 lung adenocarcinoma. Thus, the expression of these receptors may influence the stromal invasion and prognosis in pT1 lung adenocarcinoma. Further investigation will be needed to clarify the detailed relationships between the stromal invasion, prognosis and the expression of these receptors.

A complex network of chemokines and their receptors influences the development of the primary tumors and their metastatic foci. There are two aspects to the biological role of chemokines in these processes: their role in controlling leukocyte infiltration in cancer, and their influence on the metastatic potential and site-specific spread of tumor cells (35). It has been demonstrated that CCL21 and CXCL10 exert antitumor activities by inducing immune-stimulating and angiostatic effects $(36,37)$. Notably, CCL21 and CXCL10 play additional roles in the tumor micro-environment $(17,18,24,38,39)$.

In conclusion, these findings and those of previous studies suggest that CCR7 and CXCR3 play a critical role in lung adenocarcinoma cell metastasis to lymph nodes by inducing cell migration. Recently, several antagonists of chemokine receptors have been identified $(28,40)$. Our results also suggest that an antagonist of CCR7 and/or CXCR3 is useful in controlling lymph node metastasis by lung adenocarcinoma cells.

\section{References}

1. Fidler IJ: Critical factors in the biology of human cancer metastasis: Twenty-eighth G.H.A. Clowes memorial award lecture. Cancer Res 50: 6130-6138, 1990

2. Smith W and Khuri FR: The care of the lung cancer patient in the 21st century: a new age. Semin Oncol 31: 11-15, 2004.

3. Fidler IJ: Critical determinants of cancer metastasis: rationale for therapy. Cancer Chemother Pharmacol 43: 3-10, 1999. 
4. Devesa SS, Blot WJ, Stone BJ, Miller BA, Tarone RE and Fraumeni JF Jr: Recent cancer trends in the United States. J Natl Cancer Inst 87: 175-182, 1995.

5. Fidler IJ and Hart IR: Biological diversity in metastatic neoplasms: origins and implications. Science 217: 998-1003, 1982.

6. Liotta LA: An attractive force in metastasis. Nature 410: 24-25, 2001.

7. Liotta LA, Saidel MG and Kleinerman J: The significance of hematogenous tumor cell clumps in the metastatic process. Cancer Res 36: 889-894, 1976.

8. Nicolson GL: Molecular mechanisms of cancer metastasis: tumor and host properties and the role of oncogenes and suppressor genes. Curr Opin Oncol 3: 75-92, 1991.

9. Chambers AF, Groom AC and MacDonald IC: Dissemination and growth of cancer cells in metastatic sites. Nat Rev Cancer 2: 563-572, 2002.

10. Takanami I: Overexpression of CCR7 mRNA in non-small cell lung cancer: correlation with lymph node metastasis. Int J Cancer 105: 186-189, 2003.

11. Gunn MD, Kyuwa S, Tam C, Kakiuchi T, Matsuzawa A, Williams LT and Nakano H: Mice lacking expression of secondary lymphoid organ chemokine have defects in lymphocyte homing and dendritic cell localization. J Exp Med 189: 451-460, 1999.

12. Müller A, Homey B, Soto $\mathrm{H}$, et al: Involvement of chemokine receptors in breast cancer metastasis. Nature 410: 50-56, 2001.

13. Rossi D and Zlotnik A: The biology of chemokines and their receptors. Annu Rev Immunol 18: 217-242, 2000.

14. Premack BA and Schall TJ: Chemokine receptors: gateways to inflammation and infection. Nat Med 2: 1174-1178, 1996.

15. Baggiolini M: Chemokines and leukocyte traffic. Nature 392: 565-568, 1998.

16. Soto H, Wang W, Strieter RM, et al: The CC chemokine 6Ckine binds the CXC chemokine receptor CXCR3. Proc Natl Acad Sci USA 95: 8205-8210, 1998 .

17. Walser TC, Rifat S, Ma X, et al: Antagonism of CXCR3 inhibits lung metastasis in a murine model of metastatic breast cancer. Cancer Res 66: 7701-7707, 2006.

18. Kawada K, Sonoshita M, Sakashita H, et al: Pivotal role of CXCR3 in melanoma cell metastasis to lymph nodes. Cancer Res 64: 4010-4017, 2004.

19. Cyster JG: Chemokines and cell migration in secondary lymphoid organs. Science 286: 2098-2101, 1999.

20. von Andrian UH and Mempel TR: Homing and cellular traffic in lymph nodes. Nat Rev Immunol 3: 867-878, 2003.

21. Cella M, Jarrossay D, Facchetti F, et al: Plasmacytoid monocytes migrate to inflamed lymph nodes and produce large amounts of type I interferon. Nat Med 5: 919-923, 1999.

22. Janatpour MJ, Hudak S, Sathe M, Sedgwick JD and McEvoy LM: Tumor necrosis factor-dependent segmental control of MIG expression by high endothelial venules in inflamed lymph nodes regulates monocyte recruitment. J Exp Med 193: 1375-1384, 2001.

23. Martin-Fontecha A, Thomsen LL, Brett S, et al: Induced recruitment of NK cells to lymph nodes provides IFN- $\gamma$ for TH1 priming. Nat Immunol 5: 1260-1265, 2004.

24. Robledo MM, Barolom RA, Longo N, et al: Expression of functional chemokine receptors CXCR3 and CXCR4 on human melanoma cells. J Biol Chem 276: 45098-45105, 2001.
25. Sobin LH and Wittekind C (eds): TNM Classification of Malignant Tumors. 5th edition, John Wiley \& Sons, Inc., New York, 1997.

26. Sun L, Vitolo $M$ and Passaniti A: Runt-related gene 2 in endothelial cells: inducible expression and specific regulation of cell migration and invasion. Cancer Res 61: 4994-5001, 2001.

27. Kanegasaki S, Nomura Y, Nitta N, et al: A novel optical assay system for the quantitative measurement of chemotaxis. J Immunol Methods 282: 1-11, 2003.

28. Kawada K, Hosogi H, Sonoshita M, et al: Chemokine receptor CXCR3 promotes colon cancer metastasis to lymph nodes. Oncogene 26: 4679-4688, 2007.

29. Murphy PM: Chemokines and the molecular basis of cancer metastasis. N Eng J Med 345: 833-835, 2001.

30. Gunn MD, Tagemann K, Tam C, Cyster JG and Rosen SD: A chemokine expressed in lymphoid high endothelial venules promotes the adhesion and chemotaxis of naïve $\mathrm{T}$ lymphocytes. Proc Natl Acad Sci USA 95: 258-263, 1998.

31. Saeki H, Moore AM, Brown MJ and Hwang ST: Cutting edge. Secondary lymphoid-tissue chemokine (SLC) and chemokine receptor 7 (CCR7) participate in the emigration pathway of mature dendritic cells from the skin to regional lymph nodes. J Immunol 162: 2472-2475, 1999.

32. Mashino K, Sadanaga N, Yamaguchi H, et al: Expression of chemokine receptor CCR7 is associated with lymph node metastasis of gastric carcinoma. Cancer Res 62: 2937-2941, 2002.

33. Klaus G, Julia L, Golo H, Arno D, Rafael W, Werner H and Reinhold F: Prediction of lymph node metastasis in colorectal carcinoma by expression of chemokine receptor CCR7. Int $\mathbf{J}$ Cancer 116: 726-733, 2005.

34. Kawakami T, Nabeshima T, Makimoto Y, Hamasaki M, Iwasaki A, Shirakusa T and Iwasaki H: Micropapillary pattern and grade of stromal invasion in pT1 adenocarcinoma of the lung: usefulness as prognostic factors. Mod Pathol 20: 514-521, 2007.

35. Balkwill F: Cancer and the chemokine network. Nat Rev Cancer 4: $540-550,2004$

36. Tannenbaum CS, Tubbs R, Armstrong D, Finke JH, Bukowski RM and Hamilton TA: The CXC chemokine IP10and Mig are necessary for IL-12-mediated regression of the mouse RENCA tumor. J Immunol 161: 927-932, 1998.

37. Vicari AP, Ait-Yahita S, Chemin K, Mueller A, Zlotnick A and Caux C: Antitumor effects of the mouse chemokine 6Ckine/ SLC through angiostatic and immunological mechanism. J Immunol 165: 1992-2000, 2000.

38. Trentin L, Agostini C, Facco M, et al: The chemokine receptor CXCR3 is expressed on malignant B cells and mediates chemotaxis. J Clin Invest 104: 115-121, 1999.

39. Soejima K and Rollins BJ: A functional IFN-g-inducible protein-10/CXCL10-specific receptor expressed by epithelial and endothelial cells that is neither CXCR3 nor clycosaminoglycan. J Immunol 167: 6576-6582, 2001.

40. Gerlach LO, Skerlj RT, Bridger GJ and Schwartz TW: Molecular interactions of cyclam and bicyclam non-peptide antagonists with the CXCR4 chemokine receptor. J Biol Chem 276: 14153-14160, 2001. 